\title{
Hadamard Matrices of the Williamson Type
}

\author{
By L. D. Baumert and Marshall Hall, Jr.
}

1. Introduction. An Hadamard matrix is a square matrix of ones and minus ones whose row (and hence column) vectors are orthogonal. The order $n$ of an Hadamard matrix is necessarily 1,2 or $4 t$, with $t$ a positive integer. It has been conjectured that this condition ( $n=1,2$ or $4 t$ ) also insures the existence of an Hadamard matrix of that order. Constructions have been given for particular values of $n$ and even for various infinite classes of values. While other constructions exist, those given in [1]-[8] exhaust the known values of $n$. In particular, R. E. A. C. Paley [5] gave construction methods for various infinite classes of Hadamard nuatrices and indicated for each value of $n=4 t \leqq 200$ a construction which would supply an Hadamard matrix of that order with the exceptions $n=92,116,156,172,184$, 188. Since then matrices have been found for $n=92,156,172,184[1,2,7]$, leaving Paley's list still incomplete. It is interesting to note that the matrices which removed $92,156,172,184$ from the unknown category are all related to a construction given by Williamson [7]. The main purpose of this paper is to present a complete tabulation of all the known Hadamard matrices of this Williamson type for $n=4 t, t$ odd. Since an Hadamard matrix of order $2 n=2(4 t)$ can easily be constructed from one of order $n$, the question of existence for all possible orders can be reduced to the case where $t$ is odd. Thus, it is interesting to note that Hadamard matrices having the additional structure imposed by Williamson exist for all odd values of $t \leqq 27$ and, in particular, that this includes every value of $t$ for which an exhaustive search has been performed.

2. Williamson Type and Related Hadamard Matrices. An Hadamard matrix which has the form,

$$
H=\left|\begin{array}{rrrr}
A & B & C & D \\
-B & A & -D & C \\
-C & D & A & -B \\
-D & -C & B & A
\end{array}\right|,
$$

is said to be of the quaternion type, since, using the quaternion units,

$$
\begin{gathered}
e=\left|\begin{array}{llll}
1 & 0 & 0 & 0 \\
0 & 1 & 0 & 0 \\
0 & 0 & 1 & 0 \\
0 & 0 & 0 & 1
\end{array}\right|, \quad i=\left|\begin{array}{rrrrr}
0 & 1 & 0 & 0 \\
-1 & 0 & 0 & 0 \\
0 & 0 & 0 & -1 \\
0 & 0 & 1 & 0
\end{array}\right|, \\
j=\left|\begin{array}{rrrr}
0 & 0 & 1 & 0 \\
-1 & 0 & 0 & 1 \\
0 & -1 & 0 & 0
\end{array}\right|, \quad k=\left|\begin{array}{rrrr}
0 & 0 & 0 & 1 \\
0 & 0 & -1 & 0 \\
0 & 1 & 0 & 0 \\
-1 & 0 & 0 & 0
\end{array}\right|,
\end{gathered}
$$

Received September 25, 1964. This paper presents the results of one phase of research carried out at the Jet Propulsion Laboratory, California Institute of Technology, under contract number NAS 7-100, sponsored by the National Aeronautics and Space Administration. 
we may express $H$ as $A \otimes e+B \otimes i+C \otimes j+D \otimes k$, where $\otimes$ stands for the "direct" or "Kronecker" product of matrices. If we assume that $A, B, C, D$ are symmetric matrices, the Hadamard property $H H^{T}=n I$ reduces to the equations

$$
A^{2}+B^{2}+C^{2}+D^{2}=n I
$$

and

$$
\begin{aligned}
& B A-A B+D C-C D=0 \\
& C A-A C+B D-D B=0, \\
& D A-A D+C B-B C=0 .
\end{aligned}
$$

Thus, in particular, if $A, B, C, D$ are symmetric matrices of ones and minus ones which satisfy (2.2) and which are commutative in pairs, then the associated matrix $H$ of equation (2.1) will be Hadamard. We note that any matrix of this type generates an infinite class of such matrices, specifically:

Theorem 1. Let $H$ be an Hadamard matrix of the quaternion type of order $n=4 t$, with $A, B, C, D$ symmetric matrices which are commutative in pairs. Then there exist such matrices of orders $2^{i} n$ for $i=1,2,3, \cdots$.

Proof. Let

$$
\alpha=\left|\begin{array}{ll}
A & B \\
B & A
\end{array}\right|, \quad \beta=\left|\begin{array}{rr}
A & -B \\
-B & A
\end{array}\right|, \quad \gamma=\left|\begin{array}{ll}
C & D \\
D & C
\end{array}\right|, \quad \delta=\left|\begin{array}{rr}
C & -D \\
-D & C
\end{array}\right|
$$

then $\alpha, \beta, \gamma, \delta$ are symmetric matrices which are commutative in pairs. Further, $\alpha, \beta, \gamma, \delta$ satisfy $(2.2)$, that is, $\alpha^{2}+\beta^{2}+\gamma^{2}+\delta^{2}=2 n I$. Hence,

$$
H=\left|\begin{array}{rrrr}
\alpha & \beta & \gamma & \delta \\
-\beta & \alpha & -\delta & \gamma \\
-\gamma & \delta & \alpha & -\beta \\
-\delta & -\gamma & \beta & \alpha
\end{array}\right|
$$

is an Hadamard matrix of order $2 n$. Clearly this process may be iterated to provide matrices of all the indicated orders. This theorem certainly is not significant with respect to the general existence problem for Hadamard matrices, as it has long been known that the existence of an Hadamard matrix of order $n$ implies the existence of those of orders $2^{i} n$ for $i=1,2,3, \cdots$. Its significance lies in the fact that quaternion type Hadamard matrices can be constructed for these orders also.

If we insist (as did Williamson [7]) that $A, B, C, D$ be symmetric circulants satisfying equation (2.2), we get a class of Hadamard matrices, which we call the Williamson type. Thus, Williamson type matrices form a subclass of the quaternion type Hadamard matrices. In a Williamson type matrix $A, B, C, D$ and $I$ are simultaneously diagonalizable, and applying this transformation to equation (2.2) yields the equation

$$
\left(\sum_{i=0}^{t-1} a_{i} \omega_{j}^{i}\right)^{2}+\left(\sum_{i=0}^{t-1} b_{i} \omega_{j}{ }^{i}\right)^{2}+\left(\sum_{i=0}^{t-1} c_{i} \omega_{j}{ }^{i}\right)^{2}+\left(\sum_{i=0}^{t-1} d_{i} \omega_{j}{ }^{i}\right)^{2}=4 t,
$$

which must hold for each of the $t$ th roots of unity $\omega_{j}$, where the $a_{i}, b_{i}, c_{i}, d_{i}$ comprise the first rows of $A, B, C, D$, respectively. Now, restricting ourselves to odd values of $t$ and normalizing so that $a_{0}=b_{0}=c_{0}=d_{0}=+1$, we can show [7, p. 73] 


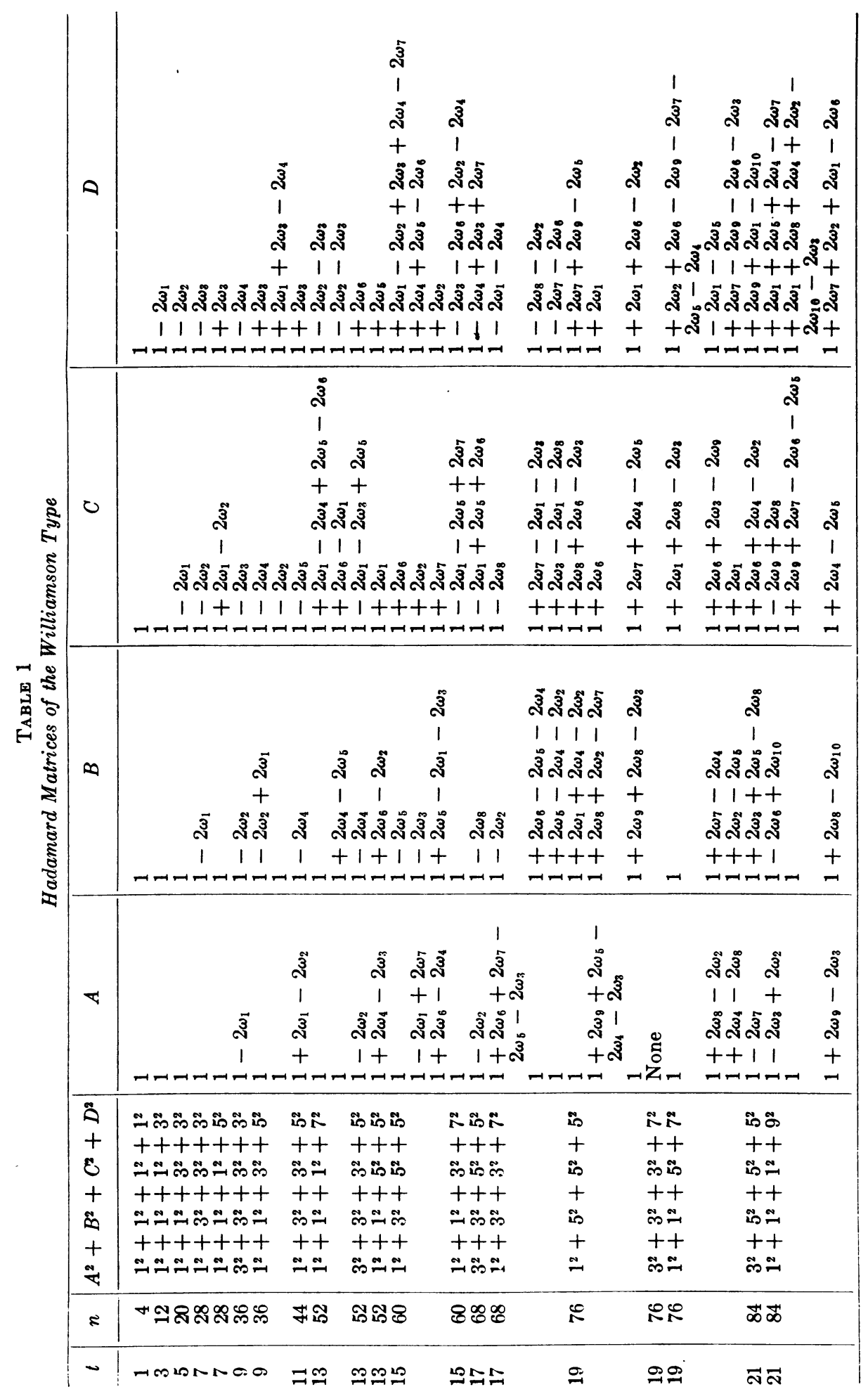




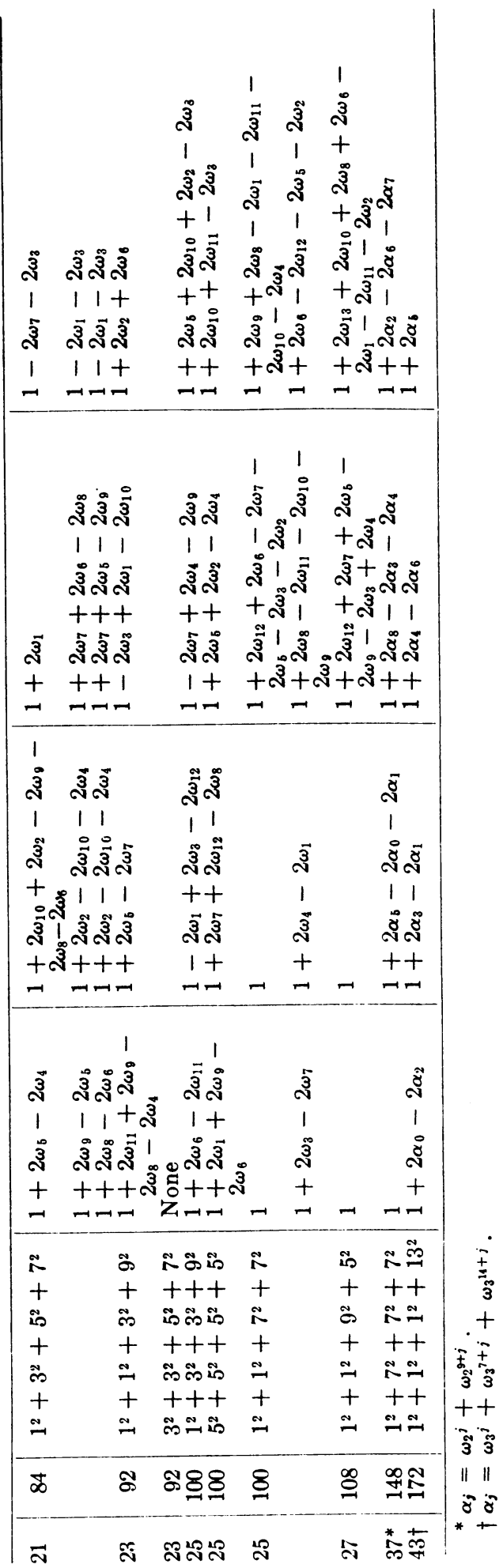




$$
H=\left|\begin{array}{rrrrrrrrrrrr}
A & A & A & B & -B & C & -C & -D & B & C & -D & -D \\
A & -A & B & -A & -B & -D & D & -C & -B & -D & -C & -C \\
A & -B & -A & A & -D & D & -B & B & -C & -D & C & -C \\
B & A & -A & -A & D & D & D & C & C & -B & -B & -C \\
B & -D & D & D & A & A & A & C & -C & B & -C & B \\
B & C & -D & D & A & -A & C & -A & -D & C & B & -B \\
D & -C & B & -B & A & -C & -A & A & B & C & D & -D \\
-C & -D & -C & -D & C & A & -A & -A & -D & B & -B & -B \\
D & -C & -B & -B & -B & C & C & -D & A & A & A & D \\
-D & -B & C & C & C & B & B & -D & A & -A & D & -A \\
C & -B & -C & C & D & -B & -D & -B & A & -D & -A & A \\
-C & -D & -D & C & -C & -B & B & B & D & A & -A & -A
\end{array}\right|
$$

Figure 1

that exactly three of the $a_{i}, b_{i}, c_{i}, d_{i}$ have the same sign, $i \neq 0$. Thus, from $a^{2}+b^{2}+c^{2}+d^{2}=4 t$, with

$$
a=\sum_{i=0}^{t-1} a_{i} \omega^{i}, \quad b=\sum_{i=0}^{t-1} b_{i} \omega^{i}, \quad c=\sum_{i=0}^{t-1} c_{i} \omega^{i}, \quad d=\sum_{i=0}^{t-1} d_{i} \omega^{i},
$$

we get

$$
\begin{aligned}
& (a+b+c-d)^{2}+(a+b-c+d)^{2} \\
& +(a-b+c+d)^{2}+(-a+b+c+d)^{2}=16 t,
\end{aligned}
$$

and, hence, a representation,

$$
T_{1}^{2}+T_{2}^{2}+T_{3}^{2}+T_{4}^{2}=4 t,
$$

where each $T_{i}$ is of the form $1 \pm 2 \omega^{j} \pm 2 \omega^{k} \pm \cdots$ and each power of $\omega$ occurs in exactly one of the $T_{i}$. Note that if we let $\omega=1$, then equation (2.4) gives us a representation of $4 t$ as the sum of 4 odd squares.

3. Table of Williamson Type Hadamard Matrices. Williamson [7] lists many solutions of equation (2.4) but does not indicate when all solutions for a particular $t$ (or for a particular representation of $4 t$ as a sum of 4 squares) is listed. For this reason we recomputed Williamson's table (in connection with the work of [1]) and extended it somewhat. In particular, all solutions for all representations of $4 t$ as a sum of 4 odd squares are given for all odd $t, 3 \leqq t \leqq 23$. In addition, some solutions are given for $t=25,27,37,43$. This exhaustive listing points up the fact that not all such representations of $4 t$ give rise to Hadamard matrices, see $t=19$ or $t=23$.

In the table the 4 squares are labeled $A, B, C, D$, respectively and $\omega_{i}=\omega^{i}+\omega^{t-i}$.

4. Paley's Exceptional Values $(92,156,172,184)$. Williamson type Hadamard matrices of orders 92 and 172 can be read from the table directly. As $184=2(92)$, an Hadamard matrix of this order can be constructed from the $H_{92}$. This is most easily done by the Kronecker product $H_{184}=H_{2} \otimes H_{92}$. However, more specialized constructions may be used, that is, we can use Theorem 1 to provide us with a quaternion type matrix or we can use Theorem 3 of Williamson [7, p. 77] to provide us with a Williamson type Hadamard matrix of this order. We note in passing that Theorem 3 of Williamson can only be applied to provide such matrices of orders $8 t$ when $t$ is odd and Williamson type solutions are known for $n=4 t$, whereas our Theorem 1 provides quaternion matrices of all orders $2^{i+2} t, i=1,2,3,: \cdots$, provided such exist for $n=4 t$. 
The remaining solution $156=3 \cdot 4 \cdot 13$ is less obvious. In [2] it is shown that an Hadamard matrix of order $12 t$ exists whenever a Williamson type matrix of order $4 t$ is known. (Actually, a quaternion type with $A, B, C, D$ symmetric and commuting in pairs will suffice.) For 156 , we use $A, B, C, D$ from a Williamson matrix of order $52=4 \cdot 13$ (see Table 1 ) and build the solution by inserting them into the matrix of Figure 1. Note that here the resulting matrix, while Hadamard, is not obviously of the quaternion type, much less a Williamson matrix.

Jet Propulsion Laboratory

California Institute of Technology

Pasadena, California

1. L. D. Baumert, S. W. Golomb \& M. Hall, JR., "Discovery of an Hadarnard matrix of order 92," Bull. Amer. Math. Soc., v. 68, 1962, pp. 237-238. MR 26 *6193.

2. L. D. BAUMERT \& MARShall Hall, JR., "A new construction for Hadamard matrices,:" Bull. Amer. Math. Soc., v. 71, 1965, pp. 169-170.

3. A. Brauer, "On a new class of Hadamard determinants," Math. Z., v. 58, 1953, pp. 219225. MR 14, 1054.

4. K. GoldBerg, "Hadamard matrices of order cube plus one," Abstract 567-90, Notices Amer. Math. Soc., v. 7, 1960 , p. 348.

5. R. E. A. C. PALEY, "On orthogonal matrices," J. Math. Phys., v. 12, 1933, pp. 311-320.

6. R. G. Stanton \& D. A. SprotT, "A family of difference sets," Canad. J. Math., v. 10, 1958, pp. 73-77. MR 19, 935 .

7. J. Williamson, "Hadamard's determinant theorem and the sum of four squares," Duke Math. J., v. 11, 1944, pp. 65-81. MR 5, 169.

8. J. Williamson, "Note on Hadamard's determinant theorem," Bull. Amer. Math. Soc., v. 53,1947, pp. $608-613$. MR 8, 559. 